\title{
DINÁMICA Y TENDENCIA DE LA EXPANSIÓN URBANA DEL GRAN CORRIENTES Y SU ÁREA DE INFLUENCIA DIRECTA
}

\section{DYNAMICS AND TENDENCY OF THE URBAN EXPANSION OF GRAN CORRIENTES AND ITS AREA OF DIRECT INFLUENCE}

Arq. López, Silvina. silvina_lopez@hotmail.com; Ing. Arce, Guillermo Antonio. gar_arce@yahoo.com.ar; Dr. Mignone, Anibal Marcelo. animarmig@hotmail.com; Dr. Alberto, Jorge Alfredo. jaalberto@hotmail.com

Centro de Geociencias Aplicadas - Facultad de Humanidades - Facultad de Ingeniería Universidad Nacional del Nordeste (UNNE)

\section{RESUMEN}

Actualmente, el sistema conformado por la ciudad de Corrientes y los centros urbanos y rurales próximos de los municipios de Santa Ana, Riachuelo, San Luis del Palmar, Paso de la Patria y San Cosme, con los que mantiene una fuerte interrelación funcional y económica, evidencia un gran desequilibrio tanto en peso poblacional como en complejidad de las actividades urbanas.

Esta situación evidencia un proceso de expansión dispersa de la ciudad Capital, donde la localización de las actividades productivas; la escasa conectividad; la incorporación de suelo periurbano y rural; los desarrollos residenciales en áreas peri-urbanas, han conformando una compleja sucesión de espacios de transición entre lo urbano y lo rural.

En este marco, el trabajo apunta a evidenciar variaciones y tendencias en la localización de la población en la capital y su área de influencia directa, relacionándolas con las formas de ocupación, los cambios de usos de suelo y el fenómeno de movilidad residencial, de manera tal que permitan visibilizar la configuración procesos de metropolización y micro regionalización, con vista a la definición de criterios para la planificación y ordenamiento territorial del espacio de estudio.

Palabras claves: Expansión Urbana Dispersa; Cambios de uso de Suelo; Espacios de Transición, Corrientes Capital

\section{ABSTRACT}

Currently, the system formed by the city of Corrientes and the nearby urban and rural centers of the municipalities of Santa Ana, Riachuelo, San Luis del Palmar, Paso de la Patria and San Cosme, with which it maintains a strong functional and economic interrelation, It shows a great imbalance in both population weight and complexity of urban activities.

This situation shows a process of dispersed expansion of the Capital city, where the location of productive activities; poor connectivity; the incorporation of peri-urban and rural land; Residential developments in peri-urban areas have shaped a complex succession of transition spaces between urban and rural areas.

In this framework, the work aims to show variations and trends in the location of the population in the capital and its area of direct influence, relating them to the forms of occupation, changes in land uses

Publicado en formato digital: López, Silvina; Arce, Guillermo Antonio; Mignone, Anibal Marcelo, Alberto, Jorge Alfredo. DINÁMICA Y TENDENCIA DE LA EXPANSIÓN URBANA DEL GRAN CORRIENTES Y SU ÁREA DE INFLUENCIA DIRECTA. Revista Geográfica Digital. IGUNNE. Facultad de Humanidades. UNNE. Año 15. № 30. Julio - Diciembre 2018. ISSN 1668-5180 Resistencia, Chaco.

En: http://hum.unne.edu.ar/revistas/geoweb/default.htm 
and the phenomenon of residential mobility, in order to make possible to visualize the configuration of metropolization and micro-regionalization processes, for defining criteria for the planning and territorial organization of the study space.

Keywords: Disperse Urban Expansion; Changes in land use; Transition Spaces, Capital of Corrientes

\section{INTRODUCCIÓN}

En las últimas décadas del siglo $\mathrm{XX}$, se produjo en el país un notorio crecimiento de las ciudades intermedias extra-pampeanas (cf. Lindenboim, 2004), fenómeno que es visible en la provincia de Corrientes. En efecto, la capital provincial muestra un importante aumento en el número de habitantes, hecho que termina por acentuar su nivel de primacía en el sistema urbano correntino. Además, cuando en el decenio de 1990, se produjo en Argentina el crecimiento de población agrupada en núcleos inferiores a los dos mil habitantes y cercanas a las grandes aglomeraciones (Leveau, 2011), este fenómeno de peri-urbanización también se replicó en el área de estudio.

Actualmente, el sistema conformado por la ciudad de Corrientes y los centros urbanos y rurales próximos de los municipios de Santa Ana, Riachuelo, San Luis del Palmar, Paso de la Patria y San Cosme, con los que mantiene una fuerte interrelación funcional y económica, evidencia un gran desequilibrio tanto en peso poblacional como en complejidad de las actividades urbanas (1).

Sobre un territorio pluri-municipal, este sistema conforma un espacio urbano físicamente discontinuo pero funcionalmente vinculado, en el que se puede identificar ámbitos específicos de organización urbana, lo que Feria (2011) caracteriza como un "área metropolitana incipiente", con una limitada materialización debido a la escasa entidad poblacional y funcional de los asentamientos que la conforman, pero con un proceso de movilidad residencial desde la ciudad central hacia su entorno circundante que constituye un factor determinante en las dinámicas espaciales metropolitanas.

En este marco, el trabajo apunta a evidenciar variaciones y tendencias en la localización de la población en la capital y su área de influencia directa, relacionándolas con las formas de ocupación, los cambios de usos de suelo y el fenómeno de movilidad residencial, de manera tal que permitan visibilizar la configuración procesos de metropolización y micro regionalización, con vista a la definición de criterios para la planificación y ordenamiento territorial del espacio de estudio.

\section{MARCO TEÓRICO Y CONTEXTUAL}

Durante los años noventa se hizo evidente un fenómeno de alto crecimiento de localidades de menos de dos mil habitantes (consideradas rurales por el INDEC), cercanas a las grandes aglomeraciones. Este fenómeno denominado peri-urbanización, fue importante durante el periodo 1991-2001, aunque ya se había producido de manera significativa en las aglomeraciones más pobladas durante la década de 1980, casos en los que el crecimiento promedio de las localidades peri-urbanas fue superior al registrado para el crecimiento promedio de las restantes localidades interiores (Leveau, 2011).

(1) El sistema urbano del Noroeste de Corrientes se ha analizado en el marco del PI H 012/14 SGCyT/UNNE, sobre la aplicación de Geoindicadores al estudio de los usos del suelo de las áreas metropolitanas del Gran Resistencia, el Gran Corrientes y sus áreas de influencia.

Publicado en formato digital: López, Silvina; Arce, Guillermo Antonio; Mignone, Anibal Marcelo, Alberto, Jorge Alfredo. DINÁMICA Y TENDENCIA DE LA EXPANSIÓN URBANA DEL GRAN CORRIENTES Y SU ÁREA DE INFLUENCIA DIRECTA. Revista Geográfica Digital. IGUNNE. Facultad de Humanidades. UNNE. Año 15. № 30. Julio - Diciembre 2018. ISSN 1668-5180 Resistencia, Chaco.

En: http://hum.unne.edu.ar/revistas/geoweb/default.htm 
En el caso de la ciudad de Corrientes, la forma de expansión discontinua se manifiesta como una compleja sucesión de espacios de transición entre lo urbano y lo rural, lo que generalmente se conoce como periurbano, rururbano y otras denominaciones "...el ámbito donde se manifiestan las hibridaciones, los aculturamientos, los modos de vida, el intercambio de flujos y el desarrollo de nuevas funciones...". (Ávila Sánchez, 2005).

Este desarrollo discontinuo, conocido como "salto de rana" o "Sprawl" (Barnes, K., Morgan, J., 2002), se caracteriza por urbanizaciones espaciadas entre sí en los márgenes del Gran Corrientes o conurbaciones en faja, como el caso de las urbanizaciones próximas a la localidades de, Santa Ana, Riachuelo, San Luis del Palmar y Paso de la Patria. En este "perímetro" en constante aumento, conviven todas las posibilidades comentadas, que traen como consecuencia demandas muy dispares y complejas, dando lugar a una variedad de problemáticas sociales, legales, económicas y ambientales, ya que este tipo de desarrollo es el que requiere de mayor inversión para poder materializarse.

Por otra parte, la intensa movilidad residencial con la pérdida de habitantes de la ciudad central en beneficio de su entorno circundante según Feria (2011) constituye un factor determinante en el alcance y conformación de las dinámicas espaciales metropolitanas, no sólo en el sentido de que es el principal condicionante de las decisiones de los actores sociales, sino también porque es el proceso mediante el cual, en definitiva, se materializan las formas urbanas.

Las nuevas configuraciones urbanas están tomando diversas topologías territoriales: mega regiones, corredores urbanos y regiones urbanas (Habitat, 2010). Esta última, las regiones urbanas, se estructuran en torno a ciudades estratégicas que extienden su influencia en el entorno regional, aunque también puede considerarse desde este punto de vista un núcleo urbano de tamaño medio (o ciudad intermedia) que ejerce influencia sobre una región determinada y sus núcleos urbanos y rurales. (Bellet y Llop, 2000)

La ciudad metropolitana es un espacio urbano sobre un territorio pluri-municipal, un sistema de asentamientos que puede ser físicamente continuo o discontinuo pero funcionalmente vinculado entre sí como conjunto, en el que se pueden identificar ámbitos específicos de organización urbana, y en cuyo proceso de conformación se pueden reconocer fases o estadios. El caso de estudio presentaría características similares a lo que Feria (2011) identifica como "áreas metropolitanas incipientes", ámbitos en los que los procesos metropolitanos están en una fase de inicio y tienen una limitada materialización espacial debido a la escasa entidad poblacional y/o funcional del sistema de asentamientos, que resulta en procesos metropolitanos poco significativos.

\section{CONSIDERACIONES METODOLOGICAS}

El área incluida en el análisis comprende las jurisdicciones de los Municipios de Corrientes, Riachuelo, Santa Ana de los Guácaras, San Cosme, Paso de la Patria y San Luis del Palmar, con ejidos municipales colindantes, considerando que los procesos de metropolización se producen sobre territorios "pluri-municipales" y que además son los municipios los que se asocian en micro regiones para desarrollar materia de competencia propia o delegada a nivel intermunicipal y supramunicipal (2).

El análisis de la distribución de la población en el área se centra en el período comprendido entre el Censo de 1970 y el Censo de 2010, por ser la década del 70 el punto de inflexión a partir del cual se inició el gran crecimiento de las ciudades intermedias, como se plantea en revisión contextual realizada.

La disponibilidad de información y datos, sobre todo los censales, condiciono el análisis debido a que la información relativa a municipios fue publicada recién a partir del Censo del 2001, por lo que se toman los datos de los departamentos Capital (municipios Corrientes y Riachuelo), San Cosme

Publicado en formato digital: López, Silvina; Arce, Guillermo Antonio; Mignone, Anibal Marcelo, Alberto, Jorge Alfredo. DINÁMICA Y TENDENCIA DE LA EXPANSIÓN URBANA DEL GRAN CORRIENTES Y SU ÁREA DE INFLUENCIA DIRECTA. Revista Geográfica Digital. IGUNNE. Facultad de Humanidades. UNNE. Año $15 . N^{\circ} 30$. Julio - Diciembre 2018. ISSN 1668-5180 Resistencia, Chaco.

En: http://hum.unne.edu.ar/revistas/geoweb/default.htm 
(municipios San Cosme, Santa Ana y Paso de la Patria) y San Luis del Palmar (municipios San Luis del Palmar y Herlitzka). En el análisis de la evolución de la población urbana y en las localidades censales, se consideraron las localidades censales existentes desde el Censo 1970 y 1980: en el Dpto. Capital: Corrientes, Laguna Brava y Riachuelo; en el Dpto. San Luis del Palmar la localidad homónima y en el Dpto. San Cosme San Cosme, Paso de la Patria y Santa Ana ${ }^{(3)}$.

El desplazamiento de población debido a la movilidad residencial, se puede evidenciar con el registro del lugar de residencia en una fecha anterior 5 años antes de cada Censo. Este registro se inició en el Censo de 1970 aunque sin hacer referencia al municipio de origen hasta $2010^{(4)}$. En el Censo de población 2010 las variables de migración (excepto país de nacimiento) se incluyeron en el cuestionario ampliado que se aplicó en una muestra en las localidades de más de 50 mil habitantes y a la totalidad de la población de las de menos de 50 mil. Con los datos de la base de datos RETADAM del cuestionario ampliado del Censo 2010, considerando las Variables: "Donde vivía hace 5 años" y "En que municipio vivía hace 5 años", se realizó el análisis hasta el corte del área de Departamento del área geográfica Corrientes.

Por lo antes explicado, para el análisis de las migraciones inter-jurisdiccionales se tomó para el análisis el periodo 2005-2010, identificando la migración de los últimos 5 años desde otros municipios de otros departamentos, (migraciones inter-departamentales), no así desde los municipios del mismo departamento ya que la información se presenta por departamento.

\section{EL ÁREA DE INFLUENCIA DIRECTA DE CORRIENTES CAPITAL}

\section{El sistema de asentamientos}

La Capital de Corrientes, que con 343 mil habitantes (INDEC, 2010) concentra el 35\% de la población de la provincia, es una ciudad media en la que predominan las actividades vinculadas con la prestación de servicios a escala ciudad y de un entorno regional, con importante participación económica de la administración pública y los servicios sociales (PET, 2011).

La ciudad Capital mantiene una fuerte interrelación funcional y económica con los centros urbanos próximos: Santa Ana de los Guácaras, ubicada a $15 \mathrm{~km}$ al NE de la ciudad de Corrientes y Riachuelo, localizada a $18 \mathrm{~km}$ al SE de la ciudad; en un segundo "anillo", San Luis del Palmar se encuentra a 24 $\mathrm{km}$ al E de Corrientes, Paso de la Patria a $35 \mathrm{~km}$ y San Cosme a $32 \mathrm{~km}$. (Gráfico N ${ }^{\circ} 1$ ). Estas localidades con gran desequilibrio tanto en peso poblacional como en complejidad de las actividades urbanas, son cabecera de municipios con ejidos municipales colindantes, bajo cuya jurisdicción se encuentra un vasto territorio rural.

(2) Art.227 de la Constitución de la Provincia de Corrientes.

(3) No fueron consideradas por ese motivo Colonia Herlitzka y San Cayetano.

(4) Del Censo 2001 se puede obtener datos de la variable "Donde vivía hace 5 años", que permite identificar la población que vivía "En esta localidad o paraje" y la que vivía "En esta provincia pero en otra localidad o paraje", sin tener referencia del municipio de origen.

Publicado en formato digital: López, Silvina; Arce, Guillermo Antonio; Mignone, Anibal Marcelo, Alberto, Jorge Alfredo. DINÁMICA Y TENDENCIA DE LA EXPANSIÓN URBANA DEL GRAN CORRIENTES Y SU ÁREA DE INFLUENCIA DIRECTA. Revista Geográfica Digital. IGUNNE. Facultad de Humanidades. UNNE. Año 15. № 30. Julio - Diciembre 2018. ISSN 1668-5180 Resistencia, Chaco.

En: http://hum.unne.edu.ar/revistas/geoweb/default.htm 

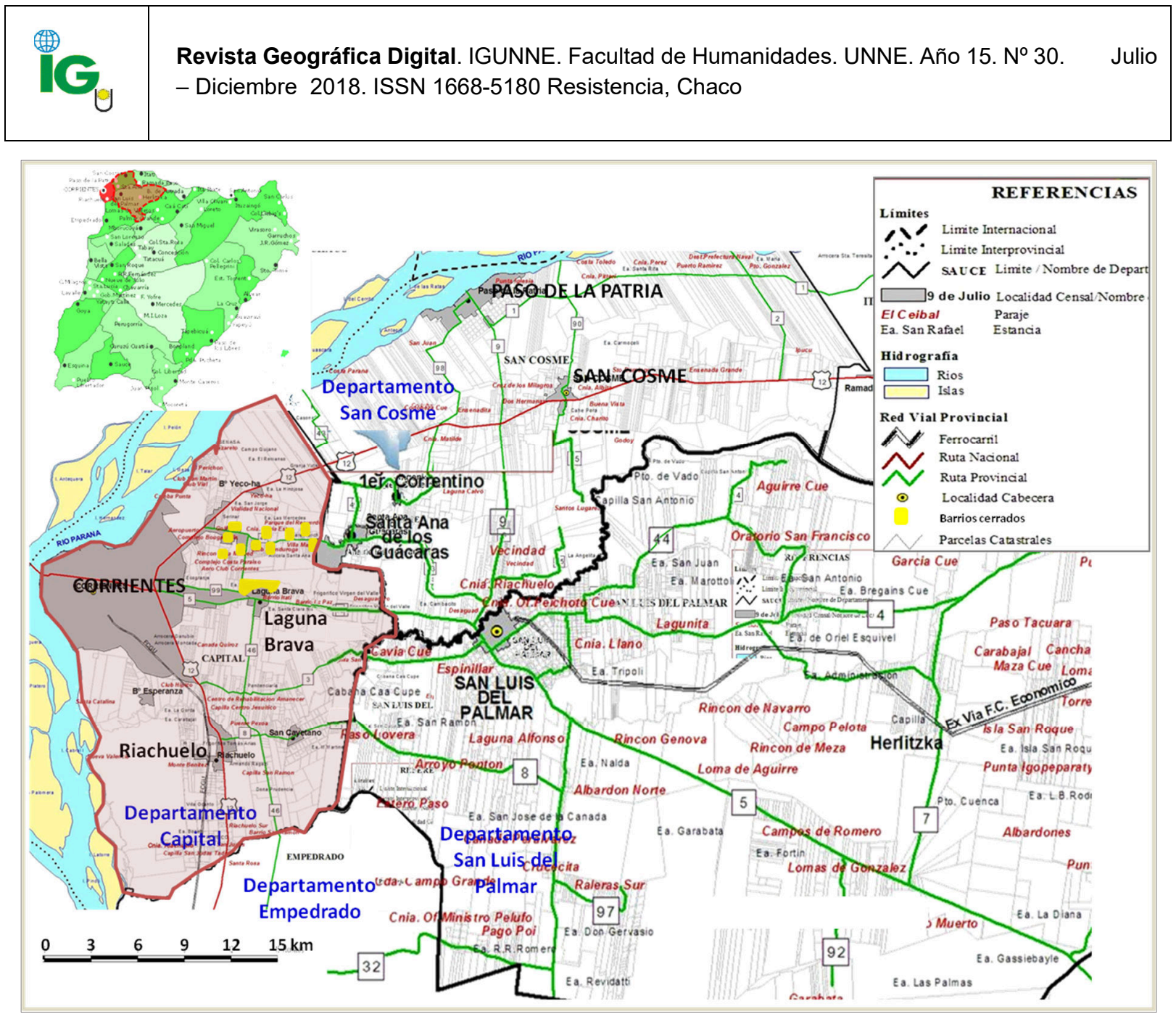

Gráfico N¹: Área de estudio. Localidades, municipios y departamentos. Fuente: elaboración propia en base a mapas de la DEyC de Corrientes

En trabajos anteriores se han realizado aproximaciones al análisis del espacio construido de la Capital y su área de influencia directa, considerando el tipo de actividades, el grado de conectividad de las redes vial y ferroviaria, la movilidad de las personas y la movilidad residencial en diferentes periodos históricos. Se puede recuperar a modo de síntesis algunas cuestiones analizadas y consideraciones realizadas, a los efectos de enmarcar el eje de análisis de este trabajo:

- La configuración del sistema urbano del NO de la Provincia de Corrientes se organizó sobre la estructura del territorio colonial, y fue condicionada por el rol marginal de este espacio en el contexto de la organización política y administrativa del territorio argentino.

- La Capital desde sus inicios fue sede administrativa y comercial, mientras que el resto del sistema de asentamientos del área ha tenido como principal función productiva la actividad agropecuaria minifundista y familiar.

- Se produjeron cambios en los usos del suelo por la localización de emprendimientos productivos y turísticos en los 80 y 90 , debido a los atractivos naturales y a la localización estratégica de estas localidades con respecto a la ciudad de Corrientes (y de Resistencia).

- La baja conectividad resultante de la estructura vial y ferroviaria, ha tenido gran influencia en el escaso desarrollo de las localidades y en la localización de las actividades en el territorio.

Publicado en formato digital: López, Silvina; Arce, Guillermo Antonio; Mignone, Anibal Marcelo, Alberto, Jorge Alfredo. DINÁMICA Y TENDENCIA DE LA EXPANSIÓN URBANA DEL GRAN CORRIENTES Y SU ÁREA DE INFLUENCIA DIRECTA. Revista Geográfica Digital. IGUNNE. Facultad de Humanidades. UNNE. Año 15. № 30. Julio - Diciembre 2018. ISSN 1668-5180 Resistencia, Chaco.

En: http://hum.unne.edu.ar/revistas/geoweb/default.htm 
- El proceso de fragmentación de parcelas rurales y el desarrollo de urbanizaciones se inició en los 90 con destino a viviendas de fin de semana y actividades turísticas. A partir del 2000 aumentaron en cantidad y tamaño, muchas de ellas con el formato de barrios cerrados.

- La incorporación de suelo urbano se produce como resultado del funcionamiento del mercado inmobiliario, en el que la rentabilidad del suelo para usos residenciales presiona sobre el uso productivo, modificando el uso de suelo a residencial.

\section{Niveles de urbanización del área}

Los asentamientos del área han tenido procesos diferenciales de crecimiento que se pueden evidenciar en la evolución de los niveles de urbanización que presentan los departamentos, considerando población urbana a la que vive en localidades de más de 2000 habitantes.

Si se analiza la evolución de la población urbana en el período 1970-2010, el Departamento Capital ya tenía más del $90 \%$ de población urbana en la década del 70 , alcanzando su máximo valor en el censo 1991 y presentando una tendencia descendente en los censos posteriores a pesar del crecimiento sostenido de la ciudad de Corrientes (Gráfico $\mathrm{N}^{\circ} 2$ ). Esto podría ser explicado por la localización y crecimiento de urbanizaciones en las áreas peri-urbanas y rurales, como se dijera anteriormente.

El Dpto. San Luis del Palmar contaba con una población urbana cercana al $50 \%$ en 1991, con un leve crecimiento en la década siguiente y próxima al 70\% para 2010. En el Dpto. San Cosme, se observan dos instancias de aumento, en 2001 por pasar la localidad de Paso de la Patria a la categoría de urbana y en 2010, por hacerlo la localidad de San Cosme. A pesar del incremento, en el último censo no llegaba al $60 \%$ de población urbana.

Más allá que en todos los departamentos analizados predomina la población urbana, existe una gran diferencia en el proceso de urbanización del Dpto. Capital con respecto a San Cosme y San Luis del Palmar en el período considerado, presentando estos últimos un inicio más tardío aunque con una tendencia más marcada desde la década del 80.

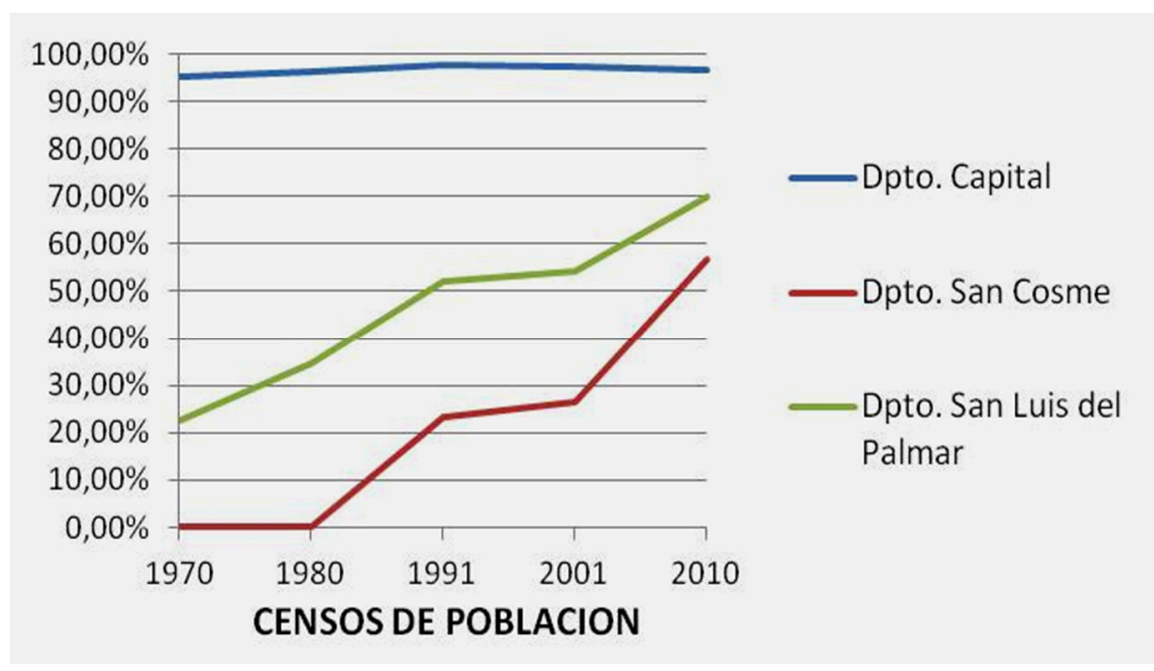

Gráfico №2: Evolución de la población urbana en los Departamentos del área (1970- 2010). Fuente: elaboración propia en base a datos de censos de población

Publicado en formato digital: López, Silvina; Arce, Guillermo Antonio; Mignone, Anibal Marcelo, Alberto, Jorge Alfredo. DINÁMICA Y TENDENCIA DE LA EXPANSIÓN URBANA DEL GRAN CORRIENTES Y SU ÁREA DE INFLUENCIA DIRECTA. Revista Geográfica Digital. IGUNNE. Facultad de Humanidades. UNNE. Año 15. № 30. Julio - Diciembre 2018. ISSN 1668-5180 Resistencia, Chaco.

En: http://hum.unne.edu.ar/revistas/geoweb/default.htm 


\section{EL PROCESO DE PERI-URBANIZACIÓN}

El área urbana de la ciudad de Corrientes representa aproximadamente un $20 \%$ de la superficie del ejido municipal, con baja densidad de ocupación (promedio de aprox. 60 habitantes por hectárea), y en el área central, emplazada en el ángulo NO de la ciudad se concentra la mayor parte de la actividad comercial, financiera, administrativa, de salud, educativa, recreativa y cultural. Por otra parte, presenta una periferia muy extensa con grandes vacíos urbanos, déficits de infraestructura y servicios y serios problemas de conectividad, debido a que a partir del área central se desarrollan corredores viales con dispersión radial y escasa conexión entre ellos.

En cuanto a la vinculación con las localidades más próximas, Riachuelo y Santa Ana y las áreas periurbanas y rurales, la red vial de circulación local de calles de tierra, se vincula en forma directa a la red principal (ruta provincial o nacional pavimentada) sin una red secundaria que haga de transición entre ambas. Esta situación condiciona el recorrido del transporte público con escaso acceso al interior de las localidades, impactando en la movilidad de las personas. (Arce et.al, 2015)

La expansión dispersa y la escasa conectividad ha presionando fuertemente sobre las actividades y la localización de la población, provocando cambios en los usos del suelo en una compleja sucesión de espacios de transición entre lo urbano y lo rural.
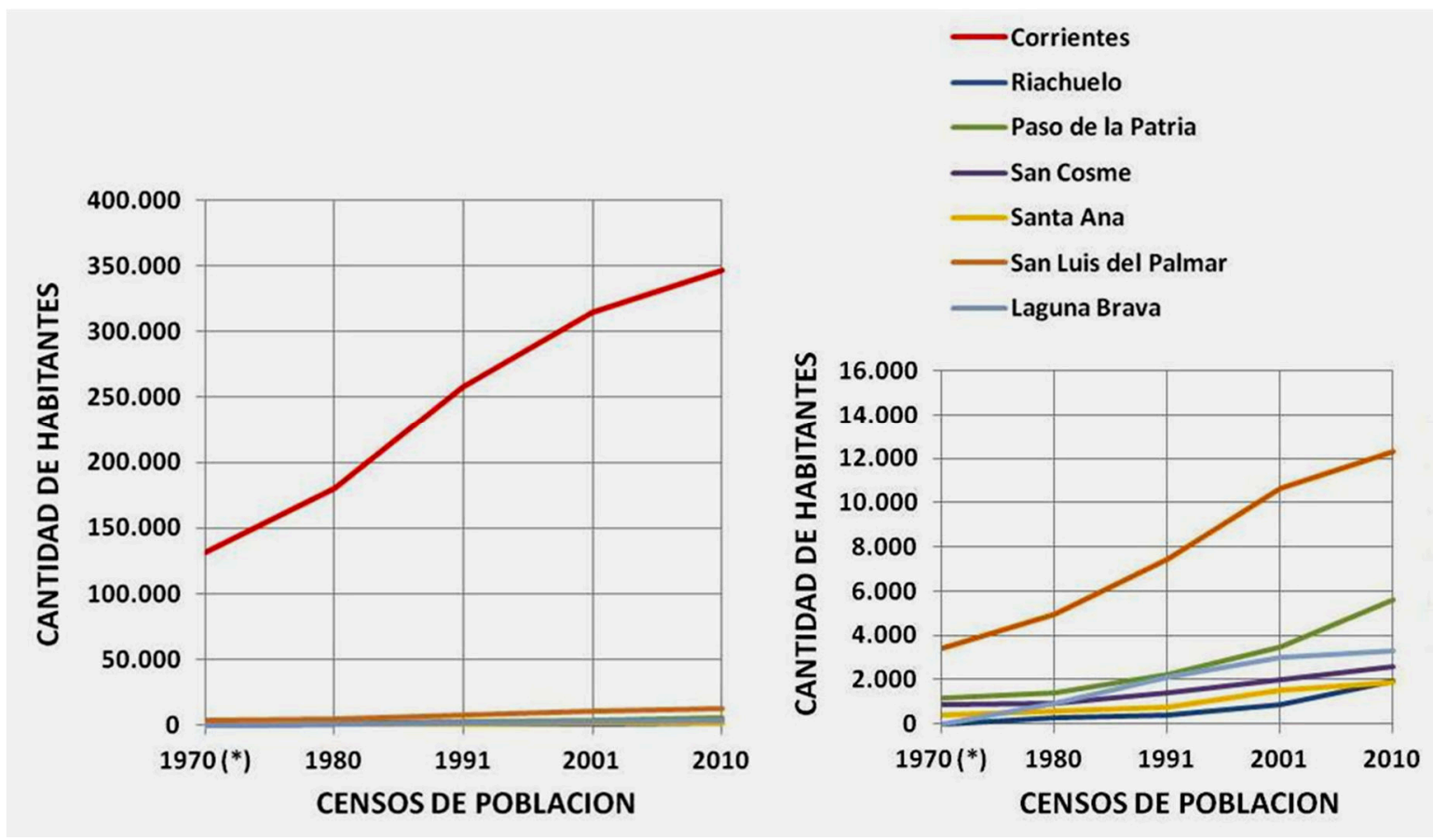

(*) el Censo de 1970 solo cuenta con datos provisorios

Gráfico №3: Evolución de la población en localidades censales del área (censos 1970 a 2010). Fuente: elaboración propia en base a Anuario 2007 y Censo Nacional de Población 2010.

Publicado en formato digital: López, Silvina; Arce, Guillermo Antonio; Mignone, Anibal Marcelo, Alberto, Jorge Alfredo. DINÁMICA Y TENDENCIA DE LA EXPANSIÓN URBANA DEL GRAN CORRIENTES Y SU ÁREA DE INFLUENCIA DIRECTA. Revista Geográfica Digital. IGUNNE. Facultad de Humanidades. UNNE. Año 15. № 30. Julio - Diciembre 2018. ISSN 1668-5180 Resistencia, Chaco.

En: http://hum.unne.edu.ar/revistas/geoweb/default.htm 


\section{Evolución de la población en las localidades}

Analizando el crecimiento poblacional de la ciudad Capital y de las localidades censales urbanas y rurales del área de estudio desde la década del 70 , se puede observar que Corrientes evidencia un incremento notable, prácticamente duplica su población entre los censos de 1970 y 1991, y luego el crecimiento se desacelera hasta llegar al $10,1 \%$ en el último período intercensal. San Luis del Palmar (considerada urbana desde 1970) es el único caso que presenta la misma tendencia que la Capital aunque con valores más elevados en todas las etapas, con mayor crecimiento entre 1980 - 1991 y una marcha más lenta entre 2001 - 2010. Con respecto a las localidades censales rurales, el mayor aumento se produce entre 1991 - 2001, y si bien los valores absolutos de población son muy pequeños, los valores relativos son importantes ya que Riachuelo creció en ese período un $126 \%$, y Santa Ana un $99 \%$. Por otra parte, la curva se suaviza en el siguiente decenio, pero los valores son mayores a Corrientes y San Luis del Palmar. (Grafico N³).

La evolución de las localidades censales en relación con el departamento que integran, permite comparar las curvas de población total de la unidad administrativa y en localidades urbanas, e indirectamente asociar con la evolución de la población rural. (Gráfico ํ4)
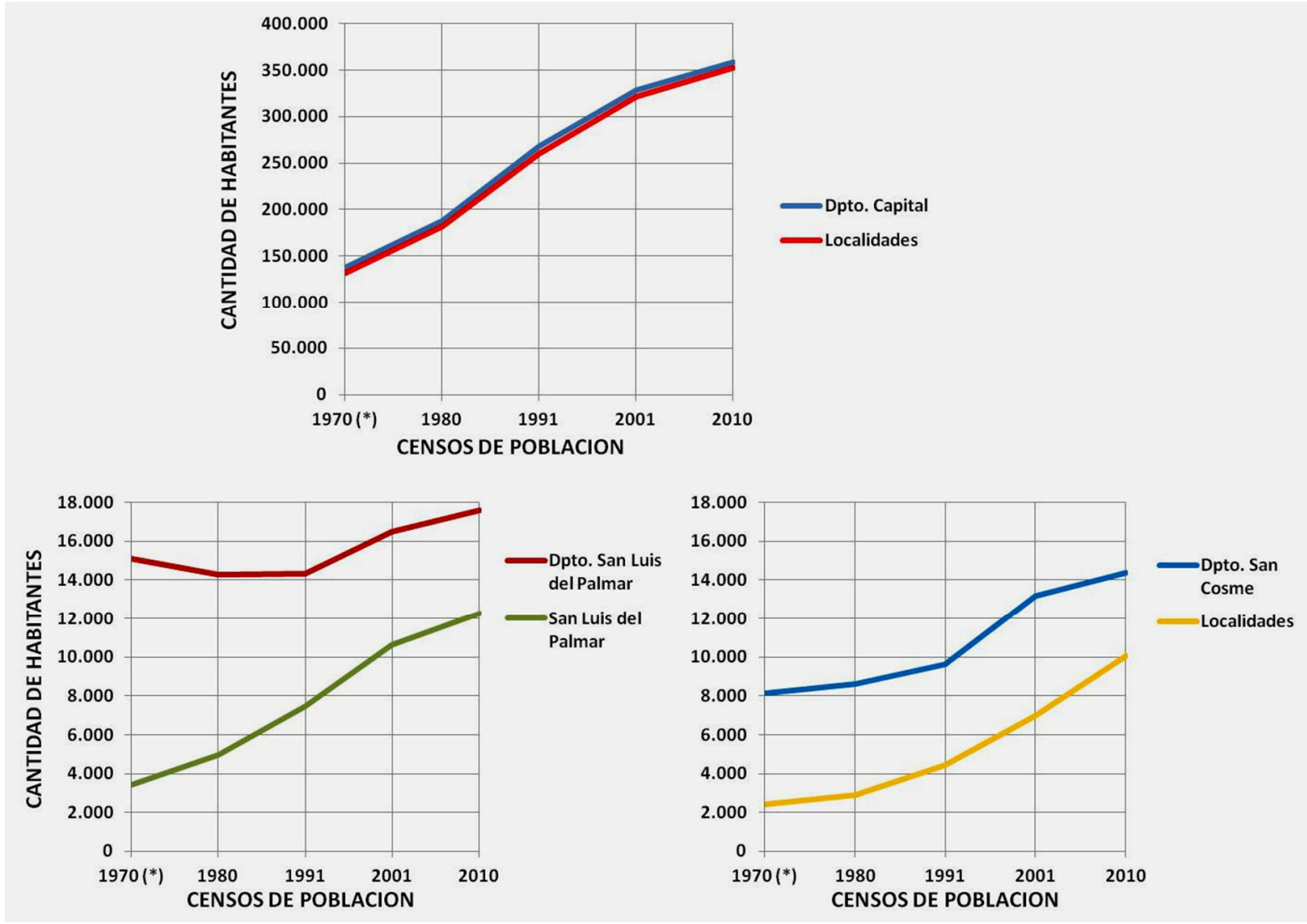

(*) el Censo de 1970 solo cuenta con datos provisorios

Gráfico N4: Comparación de la población en localidades y Departamentos (1970-2010). Fuente: elaboración propia en base a Anuario 2007 DEyC y datos de Censos 2010

Publicado en formato digital: López, Silvina; Arce, Guillermo Antonio; Mignone, Anibal Marcelo, Alberto, Jorge Alfredo. DINÁMICA Y TENDENCIA DE LA EXPANSIÓN URBANA DEL GRAN CORRIENTES Y SU ÁREA DE INFLUENCIA DIRECTA. Revista Geográfica Digital. IGUNNE. Facultad de Humanidades. UNNE. Año 15. № 30. Julio - Diciembre 2018. ISSN 1668-5180 Resistencia, Chaco.

En: http://hum.unne.edu.ar/revistas/geoweb/default.htm 
En el Dpto. Capital coinciden las curvas, porque la ciudad de Corrientes representa prácticamente la totalidad de la población del territorio. En cambio, en el Dpto. San Luis del Palmar se detecta que la localidad crece sostenidamente, mientras el departamento refleja un pendiente negativa entre $1970 \mathrm{y}$ 1991, lo que indicaría pérdida de población rural que sería absorbida por la capital departamental. Desde 1991, hay una marcha similar en las curvas, aunque la localidad crece a un ritmo mayor.

Por su parte, en el Dpto. San Cosme se observa una notoria tendencia positiva de las localidades en relación con el departamento, consecuencia del aumento en el número de habitantes de ciudades como Santa Ana y Paso de la Patria.

Con los datos presentados se puede verificar el gran crecimiento de la ciudad Capital, como ciudad intermedia entre 1970 y 1991, así como el crecimiento durante los años noventa de las localidades consideradas 'rurales' por el INDEC, próximas al aglomerado de la ciudad de Corrientes, tal como lo plantea Leveau (2011). Por otra parte, mientras que la población en localidades representa prácticamente la totalidad de la población del Dpto. Capital desde el Censo de 1970, existió una pérdida importante de población rural en el Dpto. de San Luis del Palmar entre 1970 y 1980, y en el caso del Dpto. San Cosme entre 2001 y 2010, absorbida por las localidades por otras ciudades del sistema urbano provincial o del país.

\section{EL FENÓMENO DE MOVILIDAD RESIDENCIAL}

\section{Desarrollo de urbanizaciones residenciales para población de la Capital.}

La dinámica de expansión de la ciudad que resulta en la escasa oferta de suelo con infraestructuras y equipamientos y altos valores de los inmuebles en la zona céntrica y en los barrios de mayor calidad urbana, ha provocado el incremento del número de emprendimientos inmobiliarios destinados a urbanizaciones residenciales, (la mayoría con el formato de barrios cerrados), sobre todo en los ejes de vinculación con Santa Ana y Riachuelo.

El valor de la tierra en esos proyectos es comparativamente inferior si se considera que además permite el acceso a servicios y equipamiento social y deportivo, seguridad, y hasta el uso exclusivo de lagunas y de paisajes naturales. La valorización de la propiedad inmueble al transformar tierra de uso rural en tierra de uso urbano, es una fuerte motivación para ese tipo de desarrollos, que se han incrementado en número y en tamaño y han ampliando su área de localización. (López et. al, 2015). El crecimiento de la actividad económica y comercial que se ha generado, produce el crecimiento de la demanda infraestructura y servicios, deja a cargo del estado (provincial y/o municipal) toda la carga de la construcción de los mismos, mientras que los beneficios son recogidos por los actores económicos del mercado inmobiliario.

\section{Movilidad residencial}

El traslado de la residencia de población de la Capital al área de influencia, implica pérdida de habitantes de la ciudad central en beneficio de su entorno circundante, lo que se puede evidenciar a partir de los movimientos migratorios entre las jurisdicciones. Analizando la población que migró a cada departamento en los últimos 5 años en relación con la que vivía en el mismo municipio o localidad, se puede identificar si la migración se realizó desde alguno de los municipios o localidades de los departamentos del área o de otros municipios o localidades de la Provincia.

\footnotetext{
Publicado en formato digital: López, Silvina; Arce, Guillermo Antonio; Mignone, Anibal Marcelo, Alberto, Jorge Alfredo. DINÁMICA Y TENDENCIA DE LA EXPANSIÓN URBANA DEL GRAN CORRIENTES Y SU ÁREA DE INFLUENCIA DIRECTA. Revista Geográfica Digital. IGUNNE. Facultad de Humanidades. UNNE. Año 15. № 30. Julio - Diciembre 2018. ISSN 1668-5180 Resistencia, Chaco.

En: http://hum.unne.edu.ar/revistas/geoweb/default.htm
} 
En Dpto. Capital, solo el 0,95\% de la población censada dijo "vivir hace 5 años en otro municipio o localidad de la Provincia", de los cuáles resulta escaso el número de individuos que manifestaron vivir en alguno de los municipios del área de análisis, pudiendo destacar San Luis del Palmar. En el Dpto. San Cosme, el 4,45\% de la población censada manifestó que hace 5 años vivía en "otro municipio o localidad de la Provincia", siendo significativo aquellos que residían en alguno de los municipios del área, destacándose los residentes oriundos de Corrientes. Para el caso del Dpto. San Luis del Palmar, del $1,38 \%$ de la población censada migrante, la mayoría vivía en alguno de los municipios del área, nuevamente con mayor aporte de los habitantes originarios de la jurisdicción Capital. (Gráfico №5)

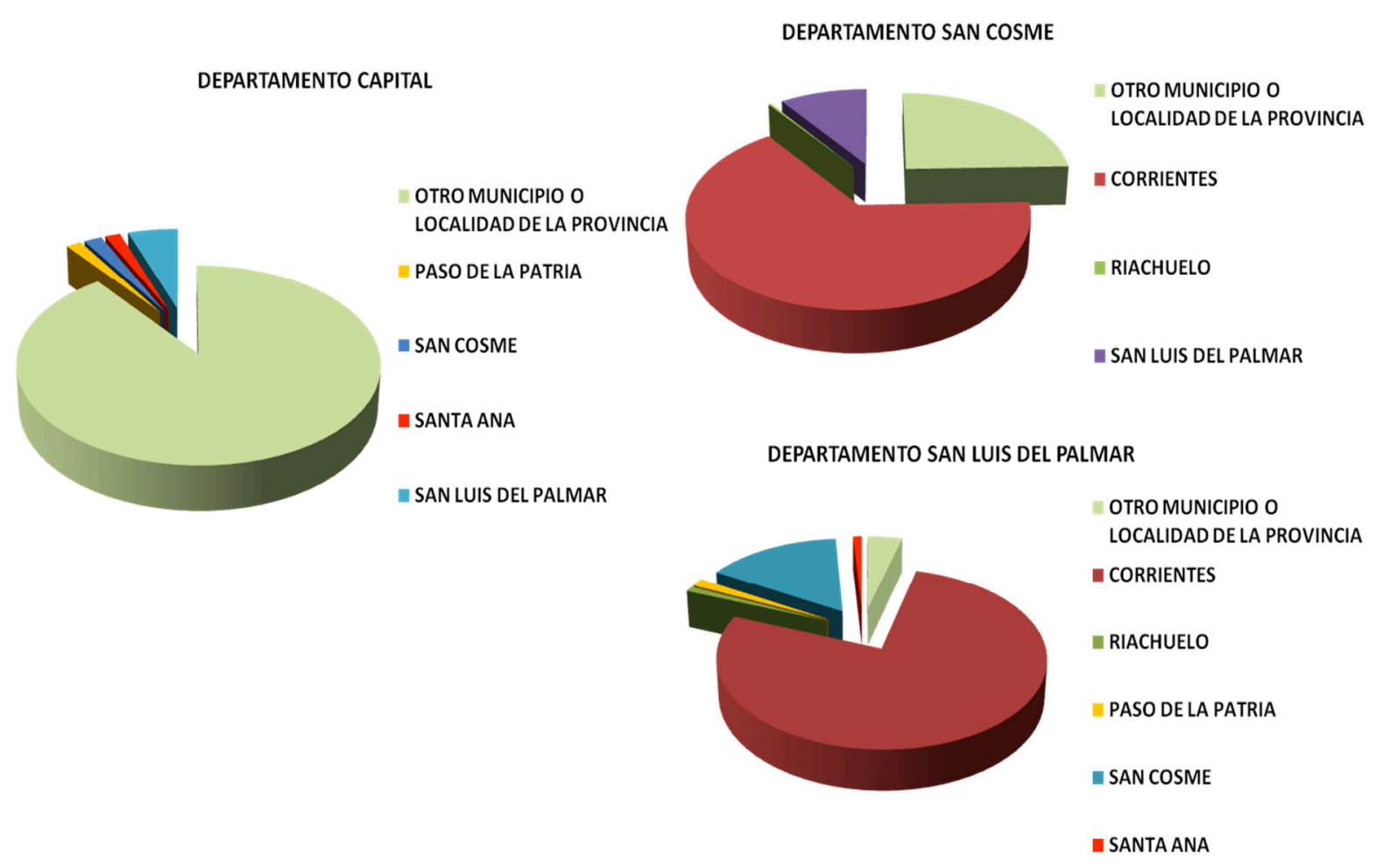

Gráfico N⒌ Población que hace 5 años vivía en otro Municipio.

Fuente: Elaboración propia con base en Censo de población 2010

Los datos analizados indican que en la migración recibida por el Dpto. Capital entre 2005 y 2010 el movimiento de población desde los municipios del área de influencia directa no es significativo en relación al proveniente de municipios o localidades de otros departamentos de la provincia. Por otra parte, en San Cosme y San Luis del Palmar la migración desde los municipios de los departamentos analizados es mayor, resaltando los originarios de Corrientes, que es la población que ha desplazado su residencia a las localidades del área de estudio.

Publicado en formato digital: López, Silvina; Arce, Guillermo Antonio; Mignone, Anibal Marcelo, Alberto, Jorge Alfredo. DINÁMICA Y TENDENCIA DE LA EXPANSIÓN URBANA DEL GRAN CORRIENTES Y SU ÁREA DE INFLUENCIA DIRECTA. Revista Geográfica Digital. IGUNNE. Facultad de Humanidades. UNNE. Año $15 . N^{\circ} 30$. Julio - Diciembre 2018. ISSN 1668-5180 Resistencia, Chaco.

En: http://hum.unne.edu.ar/revistas/geoweb/default.htm 


\section{ANTECEDENTES DE PLANIFICACIÓN TERRITORIAL MICROREGIONAL Y METROPOLITANA}

El Gran Corrientes tiene para el PET (5) el rango de "Nodo regional", y fue caracterizada como ciudad media que cumple un rol fundamental en la estructuración del territorio, en la que predominan las actividades vinculadas con la prestación de servicios a escala ciudad y de un entorno regional. Sin embargo, los centros urbanos de ese entorno no fueron considerados en el análisis del territorio realizado en el marco del Plan, que incluyó a las ciudades de más de 10.000 habitantes, rango a partir del cual se concentran funciones urbanas de relativa complejidad, según el enfoque metodológico adoptado por el mismo.

La fuerte relación de interdependencia del sistema de asentamientos urbanos y rurales y la necesidad de avanzar en la planificación del territorio, han generado varias iniciativas con relación a la identificación de un espacio micro regional de la Capital, aunque con diferentes lógicas conceptuales y metodológicas. Entre los antecedentes:

- El "Plan Estratégico para Corrientes y su Micro región de Influencia Directa" (2001), que se elaboro en cooperación entre la Municipalidad de Corrientes y la Universidad Nacional del Nordeste, incluía los municipios de Corrientes, Riachuelo, Santa Ana, San Luis del Palmar, Paso de la Patria y San Cosme. Este plan llegó a la etapa de diagnóstico participativo proponiendo una micro región basada en relaciones de proximidad y de interdependencia y aspectos potenciales para el desarrollo local en un espacio de gestión único.

- El Modelo Deseado Corrientes 2016(6) en el marco del PET (2007), propuso la regionalización de la Provincia con el criterio de dar solución a los problemas estructurales del territorio, siendo la REGION NOROESTE, la conformada por los departamentos de Capital, San Cosme, Itatí, San Luis del Palmar y Empedrado. (Grafico Nº6)

- El Plan Estratégico Participativo 2021 (2010) en el marco de la Ley N5960(7), dividió a la provincia en unidades político-administrativas definidas por la superficie del territorio, población urbana y rural y nivel de desarrollo humano. Con ese criterio se identificaron en la provincia seis regiones, denominando REGION CAPITAL al Departamento Capital (municipios de Capital y Riachuelo), dejando afuera los departamentos de San Cosme, Itatí, San Luis del Palmar y Empedrado (Grafico Nº6). Posteriormente el Modelo Deseado Corrientes para el PET fue adecuado a esta última regionalización (Grafico $\mathrm{N}^{\circ} 7$ )

Este último Plan estratégico, a diferencia de los anteriores, plantea la conformación de un territorio jurisdiccional y político que no responde a las relaciones de proximidad y de interdependencia verificadas entre la ciudad Capital y las localidades del área, ya que al realizar una escisión administrativa del sistema de asentamientos, dificulta la planificación integral de un espacio que presenta fluida entidad funcional.

(5) Análisis del sistema urbano nacional. Plan Estratégico Territorial (PET) Avance II: Argentina Urbana. Ministerio de Planificación Federal, Inversión Pública y Servicios. 2011.

(6) Secretaría de Planeamiento de la Provincia de Corrientes en el marco del Plan Estratégico Territorial, 2007.

(7) Art. 39 de la Ley № 5960 de 2010, determina que el Poder Ejecutivo debe dividir a la provincia de Corrientes en no menos de cinco regiones, en cada una de las cuales funcionará un Consejo Regional.

Publicado en formato digital: López, Silvina; Arce, Guillermo Antonio; Mignone, Anibal Marcelo, Alberto, Jorge Alfredo. DINÁMICA Y TENDENCIA DE LA EXPANSIÓN URBANA DEL GRAN CORRIENTES Y SU ÁREA DE INFLUENCIA DIRECTA. Revista Geográfica Digital. IGUNNE. Facultad de Humanidades. UNNE. Año 15. № 30. Julio - Diciembre 2018. ISSN 1668-5180 Resistencia, Chaco.

En: http://hum.unne.edu.ar/revistas/geoweb/default.htm 


\begin{tabular}{|l|l|}
\hline & $\begin{array}{l}\text { Revista Geográfica Digital. IGUNNE. Facultad de Humanidades. UNNE. Año 15. No } 30 . \\
\text { - Diciembre 2018. ISSN 1668-5180 Resistencia, Chaco }\end{array}$ \\
\hline
\end{tabular}

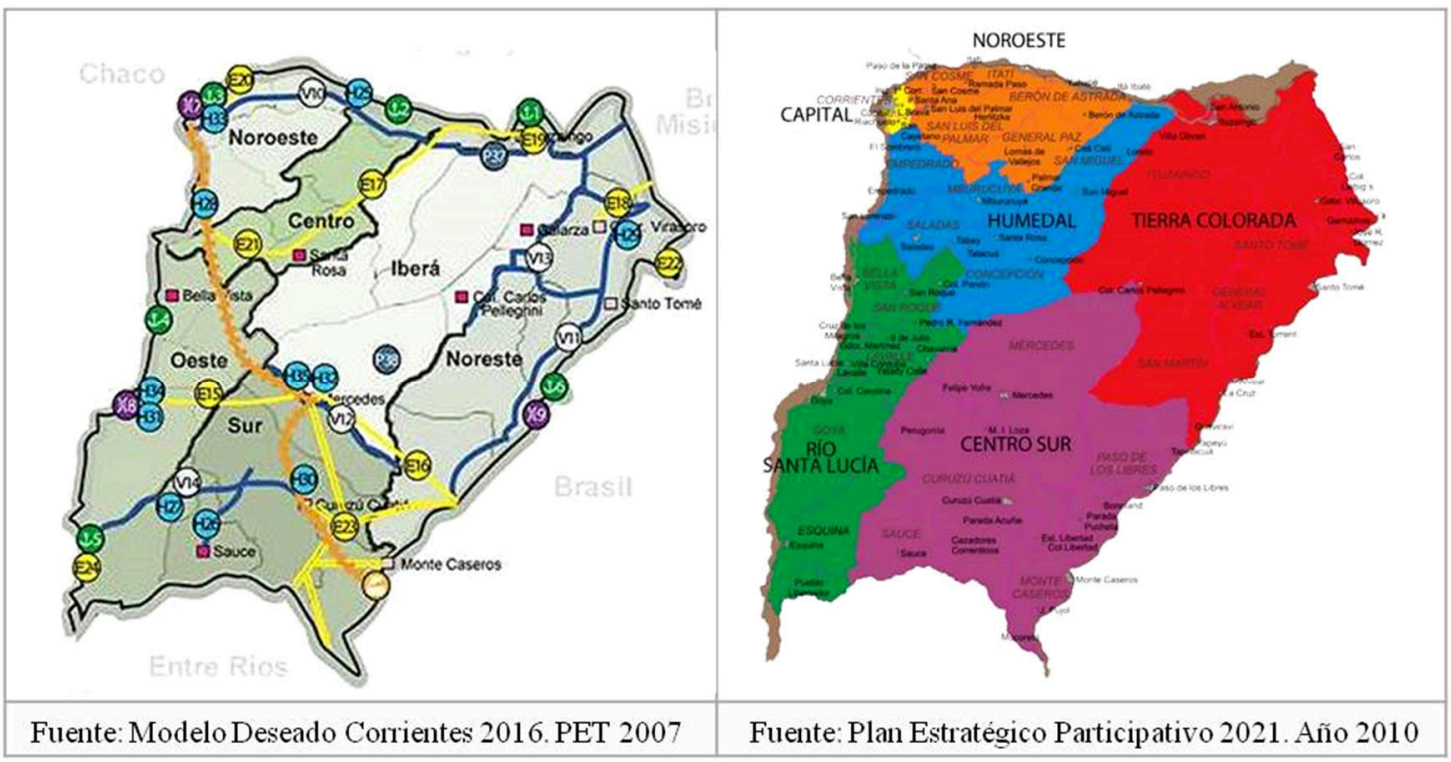

Gráfico $N^{\circ} 6$. Regionalización propuesta en los Planes



Fuente: Modelo deseado Corrientes. Subsecretaría de Planificación Territorial de la Inversión Pública. 2016

Gráfico N7. Regionalización adoptada en la actualización del PET

Publicado en formato digital: López, Silvina; Arce, Guillermo Antonio; Mignone, Anibal Marcelo, Alberto, Jorge Alfredo. DINÁMICA Y TENDENCIA DE LA EXPANSIÓN URBANA DEL GRAN CORRIENTES Y SU ÁREA DE INFLUENCIA DIRECTA. Revista Geográfica Digital. IGUNNE. Facultad de Humanidades. UNNE. Año 15. № 30. Julio - Diciembre 2018. ISSN 1668-5180 Resistencia, Chaco.

En: http://hum.unne.edu.ar/revistas/geoweb/default.htm 
Con respecto al área metropolitana de Corrientes, no está constituida ni política, ni jurídica, ni administrativamente, aunque hay antecedentes en relación con el uso genérico de "Gran Corrientes" para designarla. Desde 2001 el Instituto Nacional de Estadísticas y Censos (INDEC), considera Gran Corrientes, a la ciudad de Corrientes Capital y a las localidades del Municipio Capital (Laguna Brava y $\mathrm{B}^{\circ}$ Esperanza), aunque en los últimos años y con mayor frecuencia, está siendo identificado como Gran Corrientes el aglomerado formado por dichas localidades, al que se le suman las localidades cercanas de Riachuelo y Santa Ana.

\section{Marco normativo}

Tanto la Constitución de la Provincia ${ }^{(8)}$ como la Ley Orgánica de Municipios ${ }^{(9)}$, establecen las competencias de los niveles jurisdiccionales del Estado en la planificación del territorio. La Constitución dice en el Art. 219: "Los municipios tienen el derecho de establecer su propio orden normativo mediante el dictado de Cartas Orgánicas sancionadas por una Convención Municipal, (...). Mientras los municipios no dicten sus Cartas Orgánicas se rigen por la Ley Orgánica de Municipalidades. " y en el Art. 62: "La Provincia y los municipios, en el marco de sus respectivas competencias, ordenan el uso del suelo y regulan el desarrollo urbano, suburbano y rural. (...). La Ley Orgánica de Municipios no solo es aplicable a los municipios que carezcan de Carta Orgánica, sino que regirá en materias no regladas por estas o con carácter supletorio e interpretativo como base común del derecho público municipal de la Provincia de Corrientes. También será aplicable en cuestiones de carácter provincial, intermunicipal o que excedan el ámbito local.

La Constitución establece (Art. 227) que los municipios pueden crear micro regiones para desarrollar materia de competencia propia o delegada a nivel intermunicipal y supramunicipal, y que la participación es voluntaria.

En el mismo sentido, la Ley Orgánica de Municipios en el Art. 7 establece que los municipios pueden suscribir convenios y realizar acciones conjuntas con otros municipios en forma permanente o transitoria, de conformidad con el Art. 227 de la Constitución Provincial. Asimismo pueden asociarse y crear personas jurídicas de derecho público, privado o mixtas con finalidades de interés común. En el Art. 8 se refiere a las Relaciones inter-jurisdiccionales, declarando prioritaria la coordinación en "temas de tránsito, medio ambiente, residuos, indicadores económicos y sociales, presupuestos y formas contables, ordenamiento territorial y de aguas, planeamiento, desarrollo económico y social, defensa civil, control de epidemias y enfermedades trasmisibles, seguridad, obras públicas de interés regional y en general toda cuestión susceptible de exceder el ámbito local". A tales fines se podrá convenir la formación de organismos de coordinación inter-jurisdiccional con las facultades que la ley o los acuerdos establezcan.

Con respecto a las Cartas Orgánicas (CO), mientras el municipio de Corrientes dictó su primer $\mathrm{CO}$ en 1994(10), el resto de los municipios lo hizo en años recientes: San Luis del Palmar en 2008, Paso de la Patria en 2009, San Cosme en 2010, Santa Ana en 2012, y Riachuelo no la ha dictado aun.

(8) Constitución de la Provincia de Corrientes. Reforma constitucional 2007.

(9) Ley Nº 6042 de 2011 que actualizo la Ley Orgánica de Municipios a la Reforma Constitucional de 2007.

(10) La última reforma de la Carta Orgánica fue realizada en 2013.

Publicado en formato digital: López, Silvina; Arce, Guillermo Antonio; Mignone, Anibal Marcelo, Alberto, Jorge Alfredo. DINÁMICA Y TENDENCIA DE LA EXPANSIÓN URBANA DEL GRAN CORRIENTES Y SU ÁREA DE INFLUENCIA DIRECTA. Revista Geográfica Digital. IGUNNE. Facultad de Humanidades. UNNE. Año $15 . N^{\circ} 30$. Julio - Diciembre 2018. ISSN 1668-5180 Resistencia, Chaco.

En: http://hum.unne.edu.ar/revistas/geoweb/default.htm 
Las CO de los municipios regulan los temas relacionados con la planificación y el ordenamiento territorial y ambiental. En el caso de Corrientes establece entre los objetivos de la Políticas Municipales (Art.14), regular el ordenamiento territorial y el crecimiento armónico de la ciudad considerando las características bio-físicas, garantizando el uso racional y eficiente socialmente justo y ambientalmente equilibrado del territorio y propender a los fines sociales de la actividad urbanística.

El Municipio de Paso de la Patria en su CO (Art. 9) se declara perteneciente a la Micro región económica, social y cultural, denominada "Del Humedal", y en ese marco podrá celebrar convenios intermunicipales tendientes la planificación conjunta sobre políticas, planes y/o gestiones de interés común y decidir y aplicar políticas, planes y/o gestiones que aseguren su desarrollo y la protección del ambiente. Corresponde al Municipio planificar el desarrollo urbano (Art.47) atendiendo a las necesidades de los vecinos y a la integración de las diferentes actividades, en un marco de equidad social, y elaborar y coordinar planes urbanísticos y edilicios tendientes a regular el desarrollo y crecimiento del Municipio integrado y articulado con su entorno local y regional.

La CO Municipal de San Luis del Palmar entre los Principios Generales y Políticas Especiales en relación con la Planificación (Art. 41) propone el planeamiento integral del desarrollo urbano y rural, en base a principios de calidad de vida y preservación del medio ambiente.

La CO de San Cosme, en relación al Ordenamiento Territorial Ambiental (Art. 42) establece que el municipio ordena el uso del suelo y regula el desarrollo urbano, suburbano y rural, bajo pautas de planificación democrática y participativa de la comunidad.

El Municipio de Santa Ana en su CO establece potestades del municipio en relación con la planificación de su territorio y entre los objetivos de la planificación estratégica (Art. 50 y 52), se propone lograr un municipio funcionalmente equilibrado, socialmente equitativo y con una participación efectiva de sus vecinos, integrado y articulado con su entorno urbano, suburbano y rural. La CO extiende el planeamiento al territorio rural, especialmente orientado a las políticas de preservación del suelo.

Como se puede observar, solo en el caso de la CO de Paso de la Patria se hace referencia a la integración del municipio a una micro región (aunque la pertenencia regional no es coincidente con ninguno de los planes antes analizados), y todas sostienen principios de preservación del medio ambiente, calidad de vida y participación democrática.

Por otra parte, en ejercicio de su autonomía, cada municipio debe elaborar los planes y aprobar la normativa por medio de sus Consejos Deliberantes (órgano legislativo), dictando Ordenanzas, que son la herramienta de regulación en materia de ordenamiento territorial.

En el caso de Corrientes, el CPU(11) zonifica el territorio del municipio tanto el área urbana como rural y regula su uso y forma de ocupación. En cuanto a los otros municipios, tienen ordenanzas que regulan específicamente el área urbana de la localidad, no así normativa que regule la ocupación de todo el territorio bajo su jurisdicción.

En síntesis, los diferentes niveles del Estado son coincidentes en cuanto a los principios generales, cuentan con marcos normativos y está definida la concurrencia de los distintos organismos responsables, sin embargo la falta de una visión sistémica hace que no se establezcan las formas de articulación micro regional y / o metropolitana de los procesos de gestión y los procedimientos involucrados en la planificación del territorio.

(11) CPU. Código de Planeamiento Urbano. Ordenanza № 1071 y modificatorias.

Publicado en formato digital: López, Silvina; Arce, Guillermo Antonio; Mignone, Anibal Marcelo, Alberto, Jorge Alfredo. DINÁMICA Y TENDENCIA DE LA EXPANSIÓN URBANA DEL GRAN CORRIENTES Y SU ÁREA DE INFLUENCIA DIRECTA. Revista Geográfica Digital. IGUNNE. Facultad de Humanidades. UNNE. Año 15. № 30. Julio - Diciembre 2018. ISSN 1668-5180 Resistencia, Chaco.

En: http://hum.unne.edu.ar/revistas/geoweb/default.htm 


\section{REFLEXIONES FINALES}

El análisis comparativo muestra que más allá que en todos los departamentos analizados predomina la población urbana, existe una gran diferencia en el proceso de urbanización del Dpto. Capital con respecto a los departamentos de San Cosme y San Luis del Palmar en el período considerado, presentando estos últimos un inicio más tardío aunque con una tendencia más marcada desde la década del 80.

Con los datos presentados se puede verificar el gran crecimiento de la ciudad Capital, como ciudad intermedia entre 1970 y 1991, así como el crecimiento durante los años noventa de las localidades consideradas 'rurales' por el INDEC, próximas al aglomerado de la ciudad de Corrientes, tal como lo plantea Leveau (2011). Por otra parte, mientras que la población en localidades representa prácticamente la totalidad de la población del Dpto. Capital desde el Censo de 1970, existió una pérdida importante de población rural en el Dpto. de San Luis del Palmar entre 1970 y 1980, y en el caso del Dpto. San Cosme entre 2001 y 2010, absorbida por las localidades por otras ciudades del sistema urbano provincial o del país.

En el proceso de expansión dispersa de la ciudad Capital, la localización de actividades productivas; la escasa conectividad; la incorporación de suelo periurbano y rural a la ciudad; los desarrollos residenciales en áreas peri-urbanas y rurales (entre otros), han presionado fuertemente sobre la localización de la población en su área de influencia directa y sobre los usos del suelo, conformando una compleja sucesión de espacios de transición entre lo urbano y lo rural.

Los movimientos migratorios entre jurisdicciones entre 2005 y 2010, muestran que el desplazamiento de población desde los municipios del área de influencia directa hacia el Dpto. Capital no es significativo en relación al proveniente de municipios o localidades de otros departamentos de la provincia. Por otra parte, en San Cosme y San Luis del Palmar la migración desde los municipios de los departamentos analizados es mayor, resaltando los originarios de Corrientes, que es la población que ha desplazado su residencia a las localidades del área de estudio.

Esta tendencia es consistente con la movilidad residencial que se puede inferir a partir de la localización de actividades y desarrollos residenciales en las localidades, el área peri-urbana y rural del área analizada, aunque debería ser verificada con otros datos complementarios.

En relación con la planificación del territorio, la regionalización propuesta por el Plan provincial vigente (PEP 2021), a diferencia de los anteriores, plantea la conformación de un territorio jurisdiccional y político que no responde a las relaciones de proximidad y de interdependencia entre la ciudad Capital y las localidades del área, ya que al realizar una escisión administrativa del sistema de asentamientos, dificulta la planificación integral de un espacio que presenta fluida entidad funcional.

Contrastando la dinámica territorial con las delimitaciones de tipo político/administrativas y el marco normativo, se pudo identificar como problemática central el desigual nivel del marco normativo. Se observa que en el caso del Municipio de Capital aparecen todos los niveles de normas (Carta Orgánica, Código de planeamiento Urbano y Ordenanzas especificas), mientras que en el resto de los municipios esto no ocurre y se producen vacios en la regulación de cuestiones territoriales sobre todo las relacionadas con las áreas rurales.

El Gran Corrientes constituye una unidad funcional, con jurisdicciones municipales no coordinadas y con atomización de autoridades de diversos niveles de gobierno. Esta cuestión es relevante en cuanto a los datos disponibles a escala metropolitana y en relación con las autoridades de aplicación de las políticas públicas.

Publicado en formato digital: López, Silvina; Arce, Guillermo Antonio; Mignone, Anibal Marcelo, Alberto, Jorge Alfredo. DINÁMICA Y TENDENCIA DE LA EXPANSIÓN URBANA DEL GRAN CORRIENTES Y SU ÁREA DE INFLUENCIA DIRECTA. Revista Geográfica Digital. IGUNNE. Facultad de Humanidades. UNNE. Año 15. № 30. Julio - Diciembre 2018. ISSN 1668-5180 Resistencia, Chaco.

En: http://hum.unne.edu.ar/revistas/geoweb/default.htm 
En síntesis, los diferentes niveles del Estado son coincidentes en cuanto a los principios generales del marco normativo. Se halla adecuadamente definida la injerencia y autoridad jurisdiccional de los distintos organismos responsables, sin embargo no se establecen las formas de articulación para la gestión y la planificación del territorio micro regional y / o metropolitano.

\section{BIBLIOGRAFÍA}

Antequera Baiget, J. (2012). Tesis doctoral: Propuesta metodológica para el análisis de la sostenibilidad regional. Josep Antequera. Universitat Politècnica de Catalunya. Institut de Sostenibilitat. Programa de doctorado en Sostenibilidad. Barcelona, España.

Arce, G, López, S, Rey, C. (2015). "Encuestas de movilidad domiciliaria: Revisión bajo perspectivas de la movilidad y aplicación en las áreas metropolitanas de Resistencia y Corrientes, Argentina", en Revista Ingeniería de Transporte Vol. 19, № 01: pp 37-53.

URL:http://www.ingenieriadetransporte.org/index.php/sochitran/issue/view/20

Barnes, K., Morgan, J. (2002). "Sprawl development: its patterns, consequences, and measurement" Center for Geographic Information Sciences, Towson University. [en línea] Junio 2010:

URL: http://pages.towson.edu/morgan/files/Sprawl_Development.pdf

Bellet C. y Llop J.M. (2000). “Ciudades Intermedias”. Editorial Milenio. Universitat de Lleida. 2000.

Cuervo González, L. (2004). "El rostro urbano de América Latina Desarrollo económico y primacía urbana en América Latina. Una visión histórico-comparativa". En: Ana Clara Torres Ribeiro (Comp.). El rostro urbano de América Latina. Buenos Aires: CLACSO, pp 77- 114.

Feria, J. M. (2011). “Ciudad y territorio: nuevas dinámicas espaciales”. En: Pujadas, Isabel et al. (Eds.) Población y Espacios urbanos. Barcelona: Departamento de Geografía Humana de la UB y Grupo de Población de la AGE. pp 13-52.

Habitat - United Nation (2010). State of the world cities. 2010-2011. Bridging de urban divide. Kenya 2010.

Janoschka, M. (2002). "El Nuevo modelo de la ciudad latinoamericana: fragmentación y privatización", en Revista EURE, vol. 28, núm. 35.

Lattes, A. (1975). "Redistribución espacial y migraciones". En: Recchini, Z; Lattes, A (ed): La población de Argentina. Buenos Aires, CICRED: 95112.

Leveau, C. M. (2011). "Urbanización y contra-urbanización en Argentina: un análisis del sistema de asentamientos". Papeles de Población, vol. 17, núm. 68, abril-junio, 2011, pp. 147-159. Universidad Autónoma del Estado de México. Toluca, México.

URL: http://www.redalyc.org/articulo.oa?id=11219270007

Lindenboim, J. y Kennedy, D. (2004). "Dinámica urbana Argentina. 1960-2001. Reconstrucción y análisis de la información necesaria". En: Documento de Trabajo №3, CEPED, Centro de Estudios sobre Población, Empleo y Desarrollo. Facultad de Ciencias Económicas, UBA.

López, S, Arce, G., Alberto, J. (2015) "Indicadores de desarrollo sostenible para espacios sujetos a la presión del crecimiento urbano. Santa Ana de los Guácaras. Corrientes". Revista Geográfica Digital. [En línea] Año 12- N²3. URL: http://hum.unne.edu.ar/revistas/geoweb/homeig0.htm

Publicado en formato digital: López, Silvina; Arce, Guillermo Antonio; Mignone, Anibal Marcelo, Alberto, Jorge Alfredo. DINÁMICA Y TENDENCIA DE LA EXPANSIÓN URBANA DEL GRAN CORRIENTES Y SU ÁREA DE INFLUENCIA DIRECTA. Revista Geográfica Digital. IGUNNE. Facultad de Humanidades. UNNE. Año 15. № 30. Julio - Diciembre 2018. ISSN 1668-5180 Resistencia, Chaco.

En: http://hum.unne.edu.ar/revistas/geoweb/default.htm 
López, S; Arce, G., Alberto, J. (2016). "Procesos territoriales relacionados con la expansión urbana. Movimientos de población y desplazamiento de usos productivos en el Gran Corrientes." en Actas del XXXVI Encuentro de Geohistoria Regional. Resistencia, Instituto de Investigaciones Geohistoricas. IGHI- Conicet - UNNE. Resistencia, 2016. pp. 378 - 388.

URL: https://iighi.conicet.gov.ar/wp-content/uploads/sites/29/2017/10/Actas-Digitales-EGHR-2016Final-web.pdf

Ministerio del Interior. Presidencia de la Nación. PEP Corrientes 2021. Diagnóstico Participativo. Consulta agosto 2017.

URL: https://www.mininterior.gov.ar/planificacion/pdf/planes-prov/CORRIENTES/Plan-EstrategicoProvincial-Corrientes-2021-Diagnostico.pdf

Ministerio de Planificación Federal, Inversión Pública y Servicios (2011). "Plan Estratégico Territorial Avance II: Argentina Urbana". 1ed. Buenos Aires: Ministerio de Planificación Federal, Inversión Pública y Servicios.

Ministerio del Interior, Obras Públicas y Vivienda. Subsecretaría de Planificación Territorial de la Inversión Pública (2016). "Modelo deseado de la Provincia de Corrientes". Consultado 08/ 2017. URL: http://sippe.planificacion.gob.ar/EditarProvinciaModeloDeseado.aspx?ld=7

Rodríguez Vignoli, J. (2011). "Migración interna y sistema de ciudades en América Latina: intensidad, patrones, efectos y potenciales determinantes, censos de la década de 2000". CEPAL - Serie Población y desarrollo N 105. Publicación de las Naciones Unidas. Naciones Unidas, julio de 2011.

\section{Información censal:}

Dirección de estadísticas y Censos. Cartografía censal de la Provincia de Corrientes. Secretaría General de la Gobernación. Gobierno de la Provincia de Corrientes

URL: http://www.deyc-corrientes.gov.ar/index.php

Dirección de estadísticas y Censos. Anuario Estadístico 2007. Secretaría General de la Gobernación. Gobierno de la Provincia de Corrientes.

URL: http://www.deyc-corrientes.gov.ar/files_provincias/374.pdf. Publicado: 21/12/ 2007. Consultado: $14 / 02 / 2017$

INDEC. Censo Nacional de Población, Hogares y Vivienda. Argentina. 2010. En:

URL: http://www.censo2010.indec.gov.ar/ . Consultado: febrero 2017

INDEC. Base de datos RETADAM. Censo 2010. Cuestionario ampliado

http://200.51.91.245/argbin/RpWebEngine.exe/PortalAction?\&MODE=MAIN\&BASE=CPV2010A\&MAI

$\mathrm{N}=$ WebServerMain.inl. Consultado: febrero 2017

Publicado en formato digital: López, Silvina; Arce, Guillermo Antonio; Mignone, Anibal Marcelo, Alberto, Jorge Alfredo. DINÁMICA Y TENDENCIA DE LA EXPANSIÓN URBANA DEL GRAN CORRIENTES Y SU ÁREA DE INFLUENCIA DIRECTA. Revista Geográfica Digital. IGUNNE. Facultad de Humanidades. UNNE. Año 15. № 30. Julio - Diciembre 2018. ISSN 1668-5180 Resistencia, Chaco.

En: http://hum.unne.edu.ar/revistas/geoweb/default.htm 